\title{
Branding online en las universidades españolas. Análisis de los valores funcionales y emocionales en sus websites corporativos
}

\author{
Ana CASTILlo DíAz \\ Universidad de Extremadura \\ acasdia@alcazaba.unex.es \\ María Victoria CARRILlo DURÁN \\ Universidad de Extremadura \\ mvcardur@alcazaba.unex.es \\ Juan Luis TATO JIMÉNEZ \\ Universidad de Extremadura \\ jltato@unex.es
}

Recibido: 07/11/2012

Aceptado: 23/01/2013

\begin{abstract}
Resumen
Este trabajo presenta un análisis de la efectividad de los websites como herramienta que utiliza la totalidad de las universidades españolas. El estudio realizado explora en qué medida son empleadas las variables relativas a la marca y las diferencias en su utilización por parte de las distintas universidades españolas. Los datos de las páginas web de todas las universidades españolas se han analizado a través de una combinación de análisis de contenido y análisis multivariable. Los resultados indican que las universidades españolas prestan más atención a los valores funcionales, relacionados con las básicas actividades de educación e investigación. Sin embargo, las instituciones que presentan un planteamiento más desarrollado de sus estrategias de comunicación y marca online van otorgando cierta relevancia a los factores de carácter emocional, destacando entre ellos la proyección internacional.

Palabras clave: branding universitario, comunicación universitaria online, marcas universitarias españolas online.

\section{Spanish Universities Online Branding. Functional and Emotional Values Online Analysis in their Corporative Websites}

\begin{abstract}
This paper shows an analysis of the websites effectiveness as a tool that is used by the totality of the Spanish universities. The study explores the variables related to the brand, in what extent they are used and the differences of use by the different institutions. Spanish universities websites has been analyzed by a combination of content and multivariable analysis. Results show that Spanish universities pay more attention to the functional values, related to the traditional activities of teaching and researching. Despite this, those institutions that have more developed communication and online branding strategies start to focus their attention to emotional factors, specially their brands' international projection.

Keywords: University branding, online university communication, Spanish universities online.

\section{Referencia normalizada}

CASTILlO DÍAZ, Ana; CARRILlO DURÁN, María Victoria; y TATO JIMÉNEZ, Juan Luis (2013): "Branding online en las universidades españolas. Análisis de los valores funcionales y emocionales en sus websites corporativos". Estudios sobre el Mensaje Periodístico. Vol. 19, Núm. especial marzo, págs.: 85-97. Madrid, Servicio de Publicaciones de la Universidad Complutense.
\end{abstract}

Sumario: 1. Introducción. 2. Marco teórico; 2.1. Concepto de marca; 2.2. Marcas universitarias; 2.3. Marcas universitarias en Internet. 3. Metodología. 4. Resultados. 5. Conclusiones. 6. Referencias bibliográficas. 


\section{Introducción}

Este trabajo presenta un análisis de la efectividad de los websites como una herramienta que emplea la totalidad de las universidades españolas para comunicar sus marcas. El estudio realizado explora las variables intrínsecas a la marca y analiza en qué medida son empleadas y las diferencias en su utilización por parte de las universidades.

A pesar de que en los últimos años el área de branding en educación superior está recibiendo una creciente atención investigadora, hay una patente escasez de estudios que analicen cómo las instituciones educativas superiores muestran sus promesas de marca a través de sus webs corporativas.

Siguiendo una metodología multipasos, se han extraído datos de las páginas web de todas las universidades españolas y se han analizado a través de una combinación de análisis de contenido y análisis multivariable.

Un estudio precedente, en el que han participado los autores, ha sido llevado a cabo en las universidades británicas (Chapleo et al., 2011). La universidad española, donde conviven un número importante de instituciones de índole pública y privada, requiere un estudio particular. Asimismo este análisis representa un segundo paso para conocer en mayor medida la situación del branding universitario online dentro del contexto europeo. Este eslabón permitirá, en un futuro, ampliar el campo de revisión y establecer posibles comparaciones entre países del contexto europeo.

Se ha decidido evaluar las webs corporativas y no los recursos de la web 2.0 (O'Reilly, 2007) como redes sociales o blogs porque la comunicación que establecen las universidades en sus webs corporativas tiene una mayor trayectoria y permite una comunicación más controlada de sus valores de marca. Hoy en día el 100\% de las universidades españolas cuenta con un portal corporativo que contribuye a difundir los valores que estas marcas ofrecen a sus stakeholders.

El estudio se centra en las universidades españolas ya que han recibido menor atención en lo que respecta a su comunicación de marca online. La mayor parte de estudios se ocupan de aspectos como la accesibilidad (Buenadicha et al., 2001) o aspectos de carácter general en la navegación (Thelwall, 2003). No obstante, la competencia, que las hace considerar sus estrategias en términos de negocio (Kotler y Kotler, 1998), fuerza a estas organizaciones a considerar su imagen internacional y sus estrategias de marca (Gray et al, 2003).

El presente trabajo trata de alcanzar dos objetivos:

- Determinar hasta qué punto las universidades españolas están transmitiendo de manera efectiva sus promesas de marca online y detectar qué factores son los más destacados por parte de las universidades españolas en su branding online.

- Clasificar las universidades españolas en función del modo en que muestran sus promesas de marca online.

En consonancia con los nuevos retos de las universidades hoy en día, los resultados indican que las universidades españolas prestan más atención a los valores funcionales, relacionados con las actividades de educación e investigación, que a los valores de índole emocional. Sin embargo, las universidades que presentan un planteamiento más desarrollado de sus estrategias de marca online van otorgando cierta re- 
levancia también a los factores de carácter emocional, destacando entre ellos la apuesta por la proyección internacional de sus marcas.

\section{Marco teórico}

Debido a la intensa competencia existente en el ámbito educacional las instituciones en todo el mundo han comenzado una gran batalla con la intención de diferenciarse y atraer potenciales públicos de interés (Chapleo, 2004; Brown y Goonawardana, 2007). Sin embargo, aunque las universidades hoy en día son más conscientes de la importancia de cómo son percibidas (Melewar y Akel, 2005), la investigación sobre la gestión de comunicación en el contexto específico de la educación superior no ha sido objeto de una gran atención investigadora (Beerli Palacio, Díaz Meneses y Pérez Pérez, 2002).

Aunque hay muchos estudios sobre branding, en realidad la literatura refleja escasos análisis del branding en el segmento de la educación superior (Waerass y Solbakk, 2008; Hemsley-Brown y Oplatka, 2006).

\subsection{Concepto de marca}

Examinando las definiciones académicas del concepto de marca es posible encontrar una aproximación en torno a dos factores (De Chernatony y Mc William, 1990 y Caldwell y Freire, 2004): factores de carácter emocional y factores de índole racional.

Por su parte, De Chernatony y Dall'Olmo Riley (1998, Pág. 427) indican que una marca es un elemento multidimensional "a multidimensional construct whereby mangers augment products or services with values and this facilitates the process by which consumers confidently recognise and appreciate these values".

El concepto de branding, en línea con esta multidimensionalidad, tiende a acercarse al concepto de "experiencia". En este sentido, una definición actual del concepto de marca podría ser la de De Chernatony (2009, Pág.104) que la concibe como un conjunto de valores que posibilitan realizar una promesa y vivir una experiencia valiosa a sus públicos. Este autor sugiere que el énfasis que en un primer momento existía en los valores de carácter racional, tiende a convertirse hoy en día en un conjunto de valores de carácter racional y emocional que permiten a los stakeholders reconocer una promesa de experiencia de la marca. La experiencia de marca puede ser entendida como la culminación de estos factores que conducen a la consecución de una marca exitosa (Villafañe, 2004; Rowley, 2004).

Moogan et al (2001) consideran que las instituciones universitarias con marcas reconocidas e información accesible y comprensible tendrán mayores oportunidades de conseguir recursos humanos y estudiantes.

Los modelos que hablan de la marca como un modelo multidimensional (Villafañe, 2004; Keller, 2000; Veloutsou, Lewis y Patton, 2004) aplicados al contexto de la universidad pueden ser explicados de la manera siguiente:

- Factores de carácter funcional: relacionados con el funcionamiento básico de la universidad para obtener calidad e innovación.

- Factores de carácter emocional: características de empatía que la marca ofrece a sus públicos. Vinculados con las personas y sus relaciones. En este sentido la 
confianza y cómo se siente la gente sobre una universidad determinada son fundamentales para su selección. También se relaciona con valores y programas vinculados al contexto de la universidad y a la responsabilidad social.

Los valores funcionales han sido aquellos a los que tradicionalmente se ha venido otorgando mayor peso. No obstante llegados a un punto en el que la competencia es intensa, los valores de índole emocional comienzan a cobrar una importancia subrayable para poder diferenciarse de otras marcas universitarias.

En este sentido Olins (2000, Pág. 53) considera que aunque el precio y la confianza han sido tradicionalmente una parte importante del branding, los factores emocionales irían en primer lugar.

\subsection{Marcas universitarias}

Con independencia del contexto, una universidad debería comunicar las dimensiones cognitivas o racionales y afectivas o emocionales de su marca. En este sentido los responsables de la comunicación y la imagen deberían comunicar los atributos de sus instituciones que hagan referencia estos dos tipos de componentes (Beerli Palacio et al, 2002).

En estudios sobre los criterios de selección de las marcas universitarias Veloutsoy, Lewis y Patton (2004) y Hsuan-FuHo y Chia Chi Hung (2008) identificaron variables que podrían estar razonablemente conectadas con valores de índole emocional y funcional. Estos valores constituyen la base del modelo para el análisis llevado a cabo en este trabajo.

\subsection{Marcas universitarias en Internet}

En el contexto de Internet el branding no es intrínsecamente distinto del branding tradicional, lo que cambia es la manera de presentación de la marca (Christoulides et al., 2006), avanzando en gran medida hacia la idea de marca-experiencia. Los websites necesitan construir experiencias de marca online y ofrecer actividades, recursos, facilidad de acceso,... Todo ello contribuye a obtener esa experiencia vivida con la marca (Rowley, 2004).

La aplicación de las redes telemáticas a las actividades de la Educación Superior está provocando importantes modificaciones, no sólo en los procesos de enseñanza sino también en las formas y sistemas de comunicación y en la relación entre la Universidad y sus públicos. Las universidades han encontrado en la Red un espacio con numerosas posibilidades para la comunicación de sus marcas (Chapleo, 2007). La información que difunden a través de los soportes online afecta a su imagen y reputación (Wæraas y Solbakk, 2008) y por ello es imprescindible que las instituciones universitarias realicen un control permanente.

\section{Metodología}

Para este estudio se ha tomado el $100 \%$ de la población: 71 universidades españolas, de las que 23 tienen titularidad privada y 48 son públicas (tabla I). Todas ellas cuentan con sede web corporativa. 
En relación con la metodología se han seguido varios pasos:

- El primer análisis desarrollado se ha centrado en el análisis contenido, reforzado o no con elementos de carácter formal. Las unidades de análisis han sido los mensajes textuales, visuales y audiovisuales expuestos en las sedes webs de las universidades privadas y públicas españolas.

- Las variables independientes son, en función de la literatura revisada, las siguientes: 1) excelencia en docencia, 2) excelencia en investigación, 3) excelencia en gestión, 4) proyección internacional, 5) entorno universitario, 6) innovación y 7) responsabilidad social corporativa.

- Los indicadores para cada variable independiente considerada son 1) la existencia o no de mensajes, 2) su ubicación, 3) su adaptación a los stakeholders, 4) su traducción, 5) el refuerzo con datos y 6) el refuerzo audiovisual.

- Tras observar cada variable la evaluación y ponderación de cada una de ellas se llevó a cabo a través de la lógica difusa (Zadeh, 1965; Chapleo et al., 2011).

- A continuación se llevó a cabo un análisis cluster, que sirve para clasificar un conjunto de individuos (universidades) en una serie de grupos no definidos a priori.

Considerando que los objetivos de esta investigación son conocer cómo se diferencian en la comunicación de marca online los distintos grupos de universidades $\mathrm{y}$, mediante un conjunto de variables independientes clasificarlas, se ha utilizado el análisis discriminante como técnica estadística apropiada pues permite asignar una universidad a un grupo definido a priori (variable dependiente) en función de una serie de características del mismo (variables independientes). Se generaron dos funciones discriminantes basadas en el lambda de Wilks y el criterio de la razón de verosimilitud (Klecka, 1980).

Tabla I. Universidades analizadas

\begin{tabular}{l} 
Universidades públicas \\
\hline 1. A CORUNA \\
\hline 2. ALCALÁ \\
\hline 3. ALICANTE \\
\hline 4. ALMERIA \\
\hline 5. AUTÓNOMA DE BARCELONA \\
\hline 6. AUTÓNOMA DE MADRID \\
\hline 7. BARCELONA \\
\hline 8. BURGOS \\
\hline 9. CÁDIZ \\
\hline 10. CANTABRIA \\
\hline 11. CARLOS III DE MADRID \\
\hline 12. CASTILLA-LA MANCHA \\
\hline 14. COMPLUTENSE DE MADRID \\
\hline 15. EXTREMA \\
\hline 16. GIRONA \\
\hline 17. GRANADA \\
\hline 18. HUELVA \\
\hline 19. ILLES BALEARS \\
\hline 20. JAÉN \\
\hline 21. JAUME I DE CASTELLÓN \\
\hline 22. LA LAGUNA \\
\hline 23. LA RIOJA \\
\hline 24. LAS PALMAS DE GRAN CANARIA \\
\hline 26. LLÓN \\
\hline 27. MÁIDA \\
\hline 28. MIGUEL HERNÁNDEZ DE ELCHE \\
\hline 3. MURCIA \\
\hline 31. OVIEDO \\
\hline 32. PABLO DE OLAVIDE \\
\hline
\end{tabular}

\begin{tabular}{|c|c|}
\hline & Universidades privadas \\
\hline \multirow{2}{*}{$\begin{array}{l}\text { 33. PAÍS VASCO/EUSKAL HERRIKO } \\
\text { UNIBERTSITATEA }\end{array}$} & 1.CATÓLICA DE ÁVILA \\
\hline & 2. CATÓLICA DE VALENCIA SAN VICENTE MÁRTIR \\
\hline 34. POLITÉCNICA DE CARTAGENA & 3. CATÓLICA SAN ANTONIO \\
\hline 35. POLITÉCNICA DE CATALUNYA & 4.DEUSTO \\
\hline 36. POLITÉCNICA DE MADRID & 5.NAVARRA \\
\hline 37. POLITÉCNICA DE VALENCIA & 6. PONTIFICIA COMILLAS \\
\hline 38. POMPEU FABRA & 7. PONTIFICIA DE SALAMANCA \\
\hline 39. PÚBLICA DE NAVARRA & 8. ABAT OLIBA CEU \\
\hline 40. REY JUAN CARLOS & 9. ALFONSO X EL SABIO \\
\hline 41. ROVIRA I VIRGILI & 10. ANTONIO DE NEBRIJA \\
\hline 42. SALAMANCA & 11. CAMILO JOSÉ CELA \\
\hline 43. SANTIAGO DE COMPOSTELA & 12. CARDENAL HERRERA-CEU \\
\hline 44. SEVILLA & 13. EUROPEA DE MADRID \\
\hline 45. VALENCIA ESTUDI GENERAL & 14. EUROPEA MIGUEL DE CERVANTES \\
\hline 46. VALLADOLID & 15. FRANCISCO DE VITORIA \\
\hline 47. VIGO & 16. INTERNACIONAL DE CATALUNYA \\
\hline 48. ZARAGOZA & 17. MONDRAGÓN UNIBERTSITATEA \\
\hline & 18. OBERTA DE CATALUNYA \\
\hline & 19. RAMÓN LLULL \\
\hline & 20. SAN JORGE \\
\hline & 21. S.E.K. \\
\hline & 22. SAN PABLO C.E.U. \\
\hline & 23. VIC \\
\hline
\end{tabular}




\section{Resultados}

Del análisis clúster mediante Método de Ward y K-Medias, se han obtenido tres clústeres que agrupan a las universidades en función de su comportamiento.

Tabla II. Valores de las variables de las universidades que componen el clúster 1 (elaboración propia)

\begin{tabular}{|l|r|r|r|r|r|}
\hline & \multicolumn{1}{|c|}{$\mathrm{N}$} & \multicolumn{1}{|c|}{ Mínimo } & \multicolumn{1}{c|}{ Máximo } & \multicolumn{1}{c|}{ Media } & \multicolumn{1}{c|}{ Desv. típ. } \\
\hline EXC. DOCENCIA & 25 & 3,4091 & 8,4091 & 5,396384 & 1,1191219 \\
GESTIÓN & 25 &, 0000 & 4,8089 &, 320352 & 1,1328291 \\
INVEST & 25 &, 0000 & 7,7092 & 4,699676 & 1,5978885 \\
ENTORNO UNIV. & 25 &, 0000 & 6,4360 & 3,300636 & 2,4292950 \\
INNOVACIÓN & 25 &, 0000 & 5,3821 & 2,507888 & 2,1512599 \\
PROYECC.INTERN. & 25 &, 0000 & 7,7092 & 4,441188 & 1,7224762 \\
RSC & 25 &, 0000 & 7,7092 & 4,501248 & 1,8090366 \\
N válido (según lista) & 25 & & & & \\
\hline
\end{tabular}

El clúster 1 lo componen universidades que están muy concienciadas de la relevancia que representa la transmisión de los valores que constituyen su promesa de marca. Este grupo concede una atención especial a la responsabilidad social corporativa dentro de los valores emocionales a destacar por las marcas.

Se trata de un conjunto de instituciones que compiten en el complicado mercado universitario actual y apuestan por la asunción de responsabilidades menos tradicionales y por la proyección internacional de su oferta.

El siguiente grupo (tabla III) obtiene valores muy bajos en las variables funcionales y también en las de tipo emocional. Ninguno de los indicadores de tipo emocional adquiere valores suficientemente altos.

Tabla III. Valores de las variables de las universidades que componen el clúster 2

\begin{tabular}{|l|r|r|r|r|l|}
\hline & \multicolumn{1}{|c|}{$\mathrm{N}$} & \multicolumn{1}{|c|}{ Mínimo } & Máximo & \multicolumn{1}{c|}{ Media } & Desv. típ. \\
\hline EXC. DOCENCIA & 20 &, 0000 & 5,1911 & 3,588180 & 1,3689257 \\
GESTIÓN & 20 &, 0000 & 3,4956 &, 430010 & 1,0804284 \\
INVEST & 20 &, 0000 & 5,2957 & 2,726160 & 1,9502352 \\
ENTORNO UNIV. & 20 &, 0000 & 3,9179 &, 834265 & 1,5098963 \\
INNOVACIÓN & 20 &, 0000 & 4,3001 &, 354790 & 1,1189582 \\
PROYECC.INTERN & 20 &, 0000 & 4,5776 & 1,849350 & 1,9658475 \\
RSC & 20 &, 0000 & 5,5270 & 1,816970 & 1,9753121 \\
N válido (según & 20 & & & & \\
lista) & & & & & \\
\hline
\end{tabular}

El clúster 3 (tabla IV) tiene los valores más altos en las variables funcionales (docencia/investigación/gestión) de los tres grupos. Pero también se dan cuenta de la importancia que tienen los valores emocionales para la transmisión de su marca en el entorno on-line. 
Tabla IV. Valores de las variables de las universidades que componen el clúster 3 (elaboración propia)

\begin{tabular}{|l|r|r|r|c|c|}
\hline & $\mathrm{N}$ & Mínimo & Máximo & Media & Desv. típ. \\
\hline EXC. DOCENCIA & 26 & 5,3821 & 10,0000 & 7,829042 & 1,0546661 \\
GESTIÓN & 26 &, 0000 & 7,0093 & 3,509108 & 2,5946487 \\
INVEST & 26 &, 0000 & 9,3001 & 6,725512 & 2,4595723 \\
ENTORNO UNIV. & 26 &, 0000 & 8,2180 & 1,647492 & 2,6117652 \\
INNOVACIÓN & 26 &, 0000 & 9,1090 & 4,771527 & 2,5845999 \\
PROYECC.INTERN & 26 &, 0000 & 9,1090 & 5,771142 & 1,9801342 \\
RSC & 26 &, 0000 & 7,8359 & 3,802562 & 2,8477690 \\
N válido (según lista) & 26 & & & & \\
\hline
\end{tabular}

Dentro de este subgrupo (valores emocionales) destacan variables como la proyección internacional y la innovación (fundamentales en este escenario).

Una vez configurados los grupos de universidades se procedió a realizar el análisis discriminante y obtuvimos resultados que a continuación se detallan. Del total de 71 universidades únicamente una se ha quedado excluida del análisis. En la tabla V se puede ver el estadístico Lambda de Wilks para cada variable que indica en todos los casos diferencias de media significativas entre los grupos de universidades.

Tabla V. Pruebas de igualdad de las medias de los grupos (elaboración propia)

\begin{tabular}{|l|r|r|r|r|r|}
\hline & \multicolumn{1}{|c|}{$\begin{array}{c}\text { Lambda de } \\
\text { Wilks }\end{array}$} & \multicolumn{1}{c|}{$\mathrm{F}$} & $\mathrm{gl1}$ & $\mathrm{g} 12$ & \multicolumn{1}{c|}{ Sig. } \\
\hline EXC. DOCENCIA &, 309 & 75,995 & 2 & 68 &, 000 \\
GESTIÓN &, 576 & 24,985 & 2 & 68 &, 000 \\
INVEST &, 610 & 21,746 & 2 & 68 &, 000 \\
ENTORNO UNIV. &, 830 & 6,953 & 2 & 68 &, 002 \\
INNOVACIÓN &, 576 & 25,053 & 2 & 68 &, 000 \\
PROYECC.INTER &, 579 & 24,681 & 2 & 68 &, 000 \\
N. & & & & & \\
RSC &, 809 & 8,044 & 2 & 68 &, 001 \\
\hline
\end{tabular}

El test M de Box (tabla VI) indica que las matrices de covarianzas de los grupos de universidades son diferentes para cada uno con respecto al total de universidades.

Tabla VI. Test M de Box. Test de Igualdad de varianzas/covarianzas de los grupos de universidades (elaboración propia)

\begin{tabular}{|lc|r|}
\hline \multicolumn{2}{|l|}{$\begin{array}{l}\text { M de Box } \\
\text { F }\end{array}$} & Aprox. \\
& gl1 & 102,090 \\
& gl2 & 56 \\
& Sig. &, 006 \\
\hline
\end{tabular}

Contrasta la hipótesis nula de que las matrices de covarianzas poblacionales son iguales. 
Los resultados han ofrecido dos funciones discriminantes significativas (tablas VII y VIII). La función 1 aporta el 87,3\% de la varianza y la función 2 el 12,7\% restante. La segunda función también contribuye significativamente a la diferenciación entre los grupos. El estadístico Lambda de Wilks indica claramente que las medias de ambas funciones en los tres grupos de universidades son diferentes.

Tabla VII. Funciones discriminantes. Autovalores (elaboración propia)

\begin{tabular}{|l|r|r|r|r|}
\hline Función & Autovalor & \% de varianza & \% acumulado & $\begin{array}{c}\text { Correlación } \\
\text { canónica }\end{array}$ \\
\hline 1 & $5,364(\mathrm{a})$ & 87,3 & 87,3 &, 918 \\
2 &, $778(\mathrm{a})$ & 12,7 & 100,0 &, 661 \\
\hline
\end{tabular}

Tabla VIII. Lambda de Wilks. Test de igualdad de las medias de las funciones discriminantes (elaboración propia)

\begin{tabular}{|c|c|c|c|c|}
\hline $\begin{array}{c}\text { Contraste de } \\
\text { las funciones }\end{array}$ & $\begin{array}{c}\text { Lambda } \\
\text { de Wilks }\end{array}$ & Chi-cuadrado & gl & Sig. \\
\hline 1 a la 2 &, 088 & 156,482 & 16 &, 000 \\
2 &, 563 & 37,110 & 7 &, 000 \\
\hline
\end{tabular}

La tabla IX refleja los coeficientes estandarizados de las funciones discriminantes canónicas es decir, los coeficientes de las variables independientes que forman la combinación lineal o función discriminante para clasificar las universidades en uno de los tres grupos. Además, esta tabla sugiere cuales son las variables más importantes en cada función discriminante. Así, destacan en la función 1 la excelencia en la docencia, la innovación, la gestión y la investigación (valores funcionales); mientras que en la función dos destacan el entorno universitario, la RSC y la proyección internacional (valores emocionales). A su vez, el entorno universitario tiene un valor negativo en el caso de la función 1 y positivo en el de la 2. La primera función parece estar ligada a las actividades básicas de la universidad y las segundas hacen mención a la relación de la misma con el entorno.

Tabla IX. Coeficientes de las variables independientes (elaboración propia)

\begin{tabular}{|l|r|r|}
\hline \multirow{2}{*}{} & \multicolumn{2}{|c|}{ Función } \\
\cline { 2 - 3 } & \multicolumn{1}{|c|}{1} & \multicolumn{1}{c|}{2} \\
\hline EXC. DOCENCIA &, 652 &,- 001 \\
GESTIÓN &, 546 &,- 372 \\
INVEST &, 216 &,- 295 \\
ENTORNO UNIV. &,- 423 &, 838 \\
INNOVACIÓN &, 598 &, 055 \\
PROYEC.INTERN. &, 348 &, 391 \\
RSC &,- 132 &, 728 \\
\hline
\end{tabular}


Finalmente, el 98,6\% de los casos han sido bien clasificados (tabla X). Este número indica que la función discriminante es muy efectiva. Solamente una universidad del grupo 3 no ha sido bien clasificada por el análisis, debiendo estar en el grupo 1 por su comportamiento de comunicación de marca online y, sin embargo, está en el grupo 3 de universidades.

Tabla X. Resultados de la clasificación de las universidades según los grupos pronosticados y el comportamiento medido de las variables de comunicación de marca online ${ }^{1}$

(elaboración propia)

\begin{tabular}{|c|c|c|c|c|c|}
\hline & \multirow{2}{*}{$\begin{array}{l}\text { Número inicial } \\
\text { de casos }\end{array}$} & \multicolumn{3}{|c|}{$\begin{array}{l}\text { Grupo de pertenencia } \\
\text { pronosticado }\end{array}$} & \multirow[b]{2}{*}{ Total } \\
\hline & & 1 & 2 & 3 & \\
\hline \multirow[t]{3}{*}{ Original Recuento } & 1 & 25 & 0 & 0 & 25 \\
\hline & 2 & 0 & 20 & 0 & 20 \\
\hline & 3 & 1 & 0 & 25 & 26 \\
\hline \multirow[t]{3}{*}{$\%$} & 1 & 100,0 &, 0 &, 0 & 100,0 \\
\hline & 2 & , 0 & 100,0 &, 0 & 100,0 \\
\hline & 3 & 3,8 &, 0 & 96,2 & 100,0 \\
\hline
\end{tabular}

\section{Conclusiones}

A la luz de los resultados obtenidos se pueden extraer las siguientes conclusiones:

Las diferencias en comunicación online de las universidades españolas vienen explicadas por una combinación de esfuerzos en variables que cubren aspectos funcionales y variables referidas a valores emocionales, distinguiéndose significativamente unos de otros.

Los dos valores funcionales tradicionalmente más destacados por parte de las instituciones universitarias, la docencia y la investigación, aunque con variaciones, continúan siendo las variables a las que las marcas universitarias españolas siguen prestando mayor atención.

Por su parte, la gestión es la variable funcional a la que menor relevancia se otorga. Teniendo en cuenta que no sólo lo que una universidad enseña e investiga, sino también cómo gestiona sus recursos, son factores a considerar en el panorama competitivo en el que se desenvuelve la universidad, el hecho de no prestar adecuada atención a esta variable supone un error no sólo para las instituciones privadas, sino también para las públicas.

Las universidades más aventajadas a la hora de transmitir su marca en el entorno online prestan atención a la innovación como una variable que otorga valor diferencial en las coordenadas en las que actualmente se desenvuelve la educación superior. El valor de la innovación, fundamental en el contexto universitario (Kjaersdam, 2001; Tirronen y Nokkala, 2009), adquiere también una gran importancia fruto, sin duda, de los procesos de Bolonia en los que se han visto embarcadas las universidades españolas (Marín, A., Trilles, I., Zamarrón, G., 2005).

\footnotetext{
${ }^{1}$ Clasificados correctamente el $98 \%$ de los casos agrupados originales
} 
Dentro de las variables emocionales identificadas en el modelo diseñado destaca el hecho de que sean variables muy relacionadas con el entorno de la universidad. La imagen del país, la región y ciudad contribuyen, en buena medida, a la imagen de la universidad. Por otra parte, las universidades abogan cada vez más por una estrecha relación con su entorno más inmediato y procuran relaciones con diferentes stakeholders (empresas, familias, etc.) a la vez que son conscientes de que están dentro de un entorno global y procuran la internacionalización.

Buscan así en su contexto inmediato (entorno universitario) un componente fundamental de las marcas universitarias españolas on-line. La RSC juega también un importante papel en la relación de la universidad con su entorno. Por último, las posibilidades de comunicación y gestión que brinda la World Wide Web se ven por las universidades españolas como una oportunidad para conseguir la proyección internacional.

Si bien es posible apreciar que los valores emocionales comienzan a cobrar relevancia en las webs universitarias, aún tienen más peso en la comunicación online los aspectos ligados al funcionamiento básico.

Por último, se ha podido contrastar que contrariamente a la suposición inicial, la investigación apunta que la titularidad no es un factor discriminante a la hora de seguir un determinado patrón en la comunicación de las marcas universitarias españolas online.

\section{Referencias bibliográficas}

BEERLI PALACIO, Asunción, DÍAZ MENESES, Gonzalo \& PÉREZ PÉREZ, Pedro (2002): "The configuration of the university image and its relationship with the satisfaction of students". Journal of Educational Administration, 40(5), 486-505.

BUENADICHA, María et al. (2002): "Las universidades españolas en el World Wide Web: aplicación de un nuevo índice de evaluación. de sitios web", en Cepade $\mathrm{n}^{\circ}$ 27: http://www.cepade.es/Ademas/revista.asp?numero=27\#\#\#\# [fecha de consulta: 8 de mayo de 2009].

CALDWELL, Nial; FREIRE, Joao R. (2004): “The differences between branding a country, a region and a city: Applying the Brand Box Model". Journal of Brand Management, 12(1), 50-61.

CHAPLEO, Christopher (2004): "Interpretation and implementation of reputation/brand management by UK university leaders". International Journal of Educational Advancement, 5(1), 7-23.

CHAPLEO, Christopher (2007): "Barriers to brand building in UK universities?". International journal of nonprofit and voluntary sector marketing, 12 (1), 23-32.

CHAPLEO, Christopher, CARRILLO DURÁN, María Victoria and CASTILLO DÍAZ, Ana (2011): "Do UK universities communicate their brands effectively through their websites?"'. Journal of Marketing for Higher Education, 21: 1, 25-46

CHRISTODOULIDES, George; DE CHERNATONY, Leslie; FURRER, Olivier; SHIU, Eric; ABIMOLA, Temi. (2006): "Conceptualising and Measuring the Equity of Online Brands". Journal of Marketing Management, 22(7/8), 700-825. 
DE CHERNATONY, Leslie \& MCWILLIAM, Gil. (1990): "Appreciating brands as assets through a two-dimensional model". Journal of Marketing Management, 9, 173-188.

DE CHERNATONY, Leslie (2009): "Towards the holy grail of defining 'brand"”. Marketing Theory, 9 (1), 101-105.

DE CHERNATONY, Leslie, DALL OLMO RILEY, Francesca, \& HARRIS, Fiona (1998): "Criteria to assess brand success". Journal of Marketing Managment, 14(7), 765-781.

FOMBRUN, Charles (1996): Reputation. Realizing Value from the Corporate Image. Boston, Harvard Business School Press.

FONDEVILA, Joan Francesc; DEL OLMO, Josep Lluís y SIERRA, Javier (2011): "Universidades privadas, ¿un subsistema diferente?: cómo desarrollan los estudiantes sus preferencias hacia la universidad". Revista de Comunicación Vivat Academia, $\mathrm{n}^{\mathrm{o}}$ 114, Marzo 2011. Madrid. Universidad Complutense, en http://www.ucm.es/info/vivataca/numeros/n114/DATOSS.htm [fecha de consulta: 10 de marzo de 2012].

GRAY, Brendam J., FAM, Kim Shyam; LLANES, Violeta (2003): "Branding universities in Asian markets". Journal of Product \& Brand Management, 12(2), 108-120.

HEMSLEY-BROWN, Jane, \& GOONAWARDANA, Shivonne (2007): "Brand harmonization on the international higher education market". Journal of Business Research, 60(9), 942-948.

HEMSLEY-BROWN, Jane; OPLATKA, Izhar (2006): "Universities in a competitive marketplace - a systematic review of the literature on higher education marketing”. International Journal of Public Sector Management, 19 (4), 316-338.

HSUAN-FU, Ho; CHIA-CHI, Hung (2008): "Marketing mix formulation for higher education: An integrated analysis employing analytic hierarchy process, cluster analysis and correspondence analysis". International Journal of Educational Management, 22 (4), 328-340.

KELLER, Kevin Lane (2000): "Building and managing corporate brand equity", en SCHULTZ, Majken, HATCH, Mary Jo; LARSEN, Mogens Holten: The expressive organization. Oxford, Oxford University Press, pp.115-229.

KJAERSDAM, Finn (2001): "Innovation in university education", en Pudlowski, Z.J. (Ed.) Conference proceedings 4th UICEE Annual Conference on engineering education; Innovation in Engineering Education. Bangkok, Monash Engineering Education Series, pp.66-68.

KLECKA, William R. (1980): Discriminant analysis. London, Sage University Paper Series Quantitative Applications in the Social Sciences, Sage Publications, London.

KOTLER, Neil; KOTLER, Philip (1998): Museum strategy and marketing: Designing missions, building audiences, generating revenues and resources. San Francisco, Jossey-Bass. 
MARÍN, Antonio, TRILLES, Irene, ZAMARRÓN, Guadalupe (eds.) (2005): Mass media y Universidad. El reto de la comunicación en las universidades. Granada, SOMEDICYT, Universidad de Granada. AKEL, Sibel. (2005): "The role of corporate identity in the higher education sector". Corporate Communications: An International Journal, 10(1), pp. 41-57.

MELEWAR, T.C., \& AKEL, Sibel. (2005). "The role of corporate identity in the higher education sector". Corporate Communications: An International Journal, 10(1), pp. 41-57.

MOOGAN, Yvonne; BARON, Steve; BAINBRIDGE, Steve (2001): "Timings and trade-offs in the marketing of higher education courses: a conjoint approach", Marketing Intelligence \& Planning, 19, (3), pp.179-187.

O'REILLY, Tim (2007): "What is Web 2.0: Design Patterns and Business Models for the Next Generation of Software". Communications \& Strategies, 65 (1), pp. 1737.

ROWLEY, Jennifer (2004): “Online branding”. Online Information Review, 28(2), pp. 131-138.

THELWALL, Mike; AGUILLO, Isidro (2003): "La salud de las webs universitarias españolas". Revista Española de Documentación Científica, 26 (3), pp. 291-305.

TIRRONEN, Jarkko; NOKKALA, Terhi (2009): "Structural Development of Finnish Universities: Achieving Competitiveness and Academic Excellence". Higher Education Quarterly, 63 (3), pp. 219-236.

VELOUTSOU, Cleopatra; LEWIS, John W.; PATTON, Robert (2004): "University selection: Information requirements and importance". The International Journal of Educational Management, 18(3), pp.160-171.

VILLAFAÑE, Justo (2004): La buena reputación. Claves del valor intangible de las empresas. Madrid, Pirámide.

WAERAAS, Arild, \& SOLBAKK, Marianne (2008): "Defining the essence of a university: Lessons from higher education branding". Higher Education, 57(4), pp. 449-462.

ZADEH, Lofti (1965): "Fuzzy sets". Information and Control, 8 (3), pp. 338-353.

Proyecto financiado por Gobierno de Extremadura y Fondos Feder.
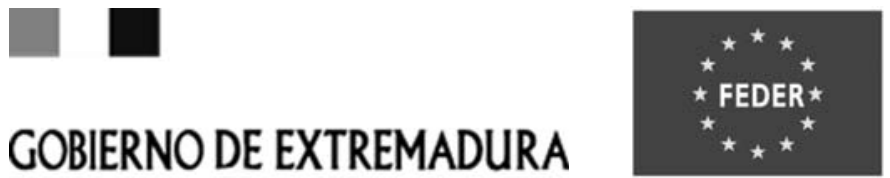

UNIÓN EUROPEA FONDO EUROPEO DE DESARROLLO REGIONAL: 


\section{Ana CASTILLO DÍAZ}

Doctora en Comunicación. Universidad de Extremadura.

Facultad de Ciencias de la Documentación y la Comunicación acasdia@alcazaba.unex.es

\section{María Victoria CARRILLO DURÁN}

Universidad de Extremadura.

Facultad de Ciencias de la Documentación y la Comunicación mvcardur@alcazaba.unex.es

\section{Juan Luis TATO JIMÉNEZ}

Universidad de Extremadura.

Facultad de Ciencias de la Documentación y la Comunicación jltato@unex.es 